\title{
SOYGRO, un modèle de simulation de la culture du soja; adaptation à des variétés cultivées dans le sud de l'Europe et validation
}

\author{
N. Brisson ${ }^{1}$, S. Bona ${ }^{2}$ et A. Bouniols 3 \\ 1 CIRAME, Hameau de Serres, 84200 Carpentras, France; \\ 2 Université de Padoue, 6 via Gradenigo, 35131 Padova, Italie; \\ з INRA, Station d'Agronomie, BP 27, 31326 Catanet-Toloson Cedex, France \\ (reçu le 21-4-1988, accepté le 28-9-1988)
}

Résumé - Le modèle SOYGRO simule la culture de différentes variétés de soja. Il est structuré en modules qui interagissent : croissance, développement, sénescence, stress hydrique, qui, à un pas de temps journalier, décrivent les principales fonctions physiologiques de la plante contribuant à l'élaboration du rendement. SOYGRO utilise des entrées concernant les techniques culturales, le climat et le sol; ce dernier étant considéré dans le modèle comme un réservoir hydrique uniquement. A l'origine, SOYGRO simulait des variétés tardives adaptées au sud des Etats-Unis; son utilisation dans le sud de l'Europe demande une adaptation à des variétés plus précoces.

L'analyse de sensibilité met en évidence l'impact des paramètres variétaux sur le résultat de la simulation et, par suite, nous renseigne sur la précision nécessaire à l'estimation de ceux-ci. Le modèle est très sensible aux paramètres phénologiques qui agissent sur la phase reproductrice du cycle, alors que les paramètres liés à l'élaboration du feuillage ont peu d'influence. La calibration concerne les variétés Weber et Kingsoy, deux variétés à croissance indéterminée, et la variété INRA 172-16 à croissance déterminée.

La validation sur des sites à climat méditerranéen montre que, pour l'ensemble des trois variétés, l'erreur de simulation est, sur le déroulement phénologique du cycle, de l'ordre de 4 à 5 jours et, de l'ordre de $3 \mathrm{q} / \mathrm{ha}$ sur le rendement. Le mode de calcul des composantes du rendement, adopté dans SOYGRO, rend difficile leur comparaison aux données observées.

croissance - Glycine $\max$ (L.) Merril - composantes du rendement - phénologie - analyse de sensibilité variétale

Summary - A soybean crop simulation model : validation and adaptation to varieties cultivated in southern Europe. The SOYGRO model simulates culture of different varieties of soybean : its structure consists of interacting modules : growth, phenology, senescence, water stress - which on a daily scale give an outline of the main physiological functions of the plant that determine yield. SOYGRO requires input data on crop, weather and soil; only the waterstorage aspect of the latter is considered in this study. SOYGRO was originally developed in the southern part of the United States to simulate late maturing varieties; its utilisation in southern Europe requires adaptation to earlier-maturing varieties.

Sensitivity analysis demonstrates the effect of varietal parameters on the model-result, and indicates the required accuracy for these parameters. The model is affected by phenological parameters which act on the reproductive phase, while those connected with leaf formation have little effect. Calibration concerns two determinate varieties, Weber and Kingsoy, and one indeterminate variety, INRA 172-16.

Validation for mediterranean climate sites showed that in the 3 varieties, phenological stages had a simulation error of 4 to 5 days, and yield an error of about $3 \mathrm{q} / \mathrm{ha}$. It is difficult to compare the SOYGRO yield components with observed data due to the method of calculation utilised.

growth - Glycine max. (L.) Merril - yield components — phenology - varietal sensitivity analysis 


\section{Introduction}

Les modèles de simulation des cultures se sont beaucoup développés ces dernières années. Les agronomes n'attendent pas seulement de ces modèles la simulation et la prévision de résultats agronomiques (rendement, matière sèche sur pied, consommation en eau, etc.), mais ils souhaitent que ces modèles soient capables de les aider à interpréter ces résultats afin d'utiliser des techniques culturales appropriées. Pour répondre à ces besoins, un nombre, plus ou moins grand selon les objectifs du modèle, de fonctions physiologiques doivent être décrites dans leur processus (Whisler et al., 1986). Chaque modèle comporte une partie mécaniste et une partie empirique; leurs importances respectives dépendent des buts recherchés, mais aussi des connaissances que l'on a du système modélisé : évolution du peuplement végétal, de la plante ou du sol.

Ces modèles se structurent autour d'un schéma de croissance : le couvert végétal intercepte le rayonnement solaire et le transforme en matière sèche grâce à son appareil assimilateur. Puis leur niveau de complexité s'accroît en même temps que le nombre de sous-modèles gravitant autour du modèle de croissance augmente.

La période de croissance peut être définie par la durée du cycle en conditions optimales pendant les périodes où les facteurs climatiques ne sont pas limitants (facteurs hydrique ou thermique), ou être issue d'un modèle phénologique mettant en jeu température et (ou) photopériode. La plupart des modèles fonctionnent avec un pas de temps journalier.

Le passage de la production de matière sèche végétale au rendement peut se faire de façon purement statistique par un indice de récolte ou par une description des processus d'élaboration du rendement tenant compte de la répartition de la matière sèche dans les différents organes de la plante.

Les modèles diffèrent également par le nombre de contraintes prises en compte. Ces contraintes, si l'on se réfère aux modèles simulant la culture du soja, sont essentiellement d'ordre thermique et hydrique (Williams et al., 1984; Wilkerson et al., 1985; Sinclair, 1986); elles peuvent être complétées par une contrainte minérale (Williams et al., 1984) ou par des aspects phytosanitaires (Wilkerson et al., 1985). L'introduction de ces contraintes nécessite la mise en cuvre de modèles parallèles : bilan hydrique, bilan minéral; attaques parasitaires.
La culture du soja s'est développée récemment en France, et plus largement en Europe. L'adaptation de cette espèce originaire de zones plus chaudes et à jours plus courts à nos conditions climatiques et culturales (hauts rendements) nécessite de bien comprendre le fonctionnement de la plante en relation avec le sol et le climat.

Les modèles de simulation de culture semblent être des outils intéressants pour répondre à ces besoins. Nous présentons, dans cet article, les principes de fonctionnement d'un modèle de croissance et de rendement d'une culture de soja, SOYGRO, élaboré par une équipe américaine de l'université de Gainsville (Etat de Floride). Ce modèle a été validé d'après les données d'essais réalisés, avec des groupes de précocité III, VII et VIII (Ecochard, 1986), dans le Sud des Etats-Unis. Sa mise en oeuvre pour le Sud de l'Europe demande donc une adaptation à des variétés plus précoces. Pour comprendre le rôle joué, dans le modèle, par les paramètres variétaux, nous présentons une analyse de sensibilité variétale, et enfin, les résultats obtenus dans un essai d'adaptation des variétés des groupes de précocité I et II dans des conditions optimales d'alimentation hydrique.

\section{Les principes de fonctionnement du modèle SOYGRO}

Le modèle SOYGRO a fait l'objet de présentations détaillées par ses auteurs (Wilkerson et al., 1983; Wilkerson et al., 1985). Nous nous contenterons ici d'en exposer les principes.

Le module de croissance (Fig. 1) constitue la trame du modèle sur laquelle les autres modules (phénologie, sénescence, stress hydrique) vont interférer. Schématiquement, il y a production d'assimilats qui sont ensuite répartis dans les divers organes de la plante (feuilles, tiges, racines, gousses et graines), ou puits, et permettent une croissance en masse. Pour le feuillage, la croissance en masse est convertie en croissance en surface, afin de décrire l'évolution de l'indice foliaire. Quand le remplissage des graines commence, la remobilisation des composés azotés, depuis les parties végétatives vers les graines, constitue une source nouvelle de matière sèche pour l'appareil reproducteur.

Le module de stress hydrique intervient sur tous les autres : quand il y a déficit hydrique, un certain nombre de fonctions vitales sont ralenties (photosynthèse, croissance de la tige, croissance en surface des feuilles, accélération de la sénescence) ou modifiées (répartition des assimilats en faveur des racines).

La phénologie régit essentiellement le schéma de répartition des assimilats, elle donne le départ pour les remobilisations azotées, participe à la croissance en surface des feuilles et intervient dans le module de sénescence. 


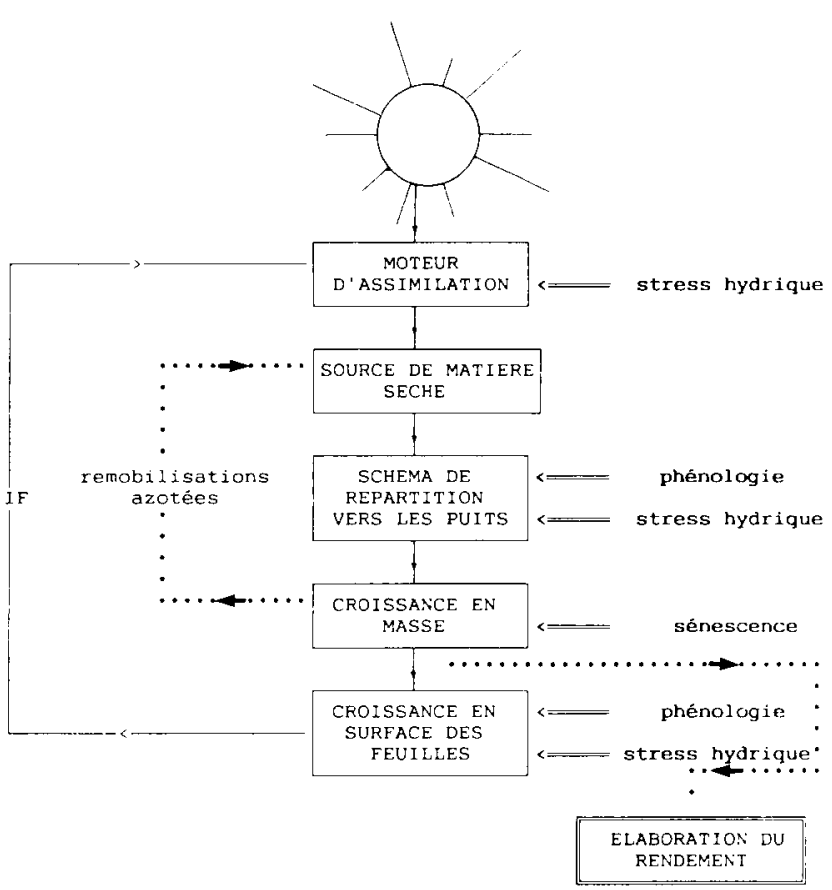

Fig. 1. Organisation du modèle SOYGRO autour du module de croissance. (...) : pendant la phase de remplissage du grain.

Les données d'entrée du modèle (Tableau I) concernent les techniques culturales, le climat et le sol. Ce dernier intervient uniquement dans le module de stress hydrique; c'est pourquoi les données d'entrée du sol sont liées à la dynamique de circulation de l'eau, dans et à la surface du sol. SOYGRO ne comporte pas de module minéral. II existe un module parasitaire qui ne sera pas détaillé ici.

\section{La phénologie}

L'échelle phénologique de SOYGRO diffère de celle de Fehr et al. (1971), par la prise en compte d'une période d'induction florale, ainsi que la fin de la croissance végétative et la fin de la floraison. De nombreux auteurs ont souligné le déterminisme thermique et photopériodique du développement du soja (Shanmugasundaram, 1978; Seddigh et Jollif, 1984; Ecochard, 1986). Ainsi, les auteurs de SOYGRO définissent deux unités journalières de développement, l'une thermique et l'autre photothermique.

\section{L'unité de développement thermique (UT)}

II s'agit d'une somme de températures horaires pondérées par des températures seuil $\left(7^{\circ} \mathrm{C}\right.$ au minimum, $30^{\circ} \mathrm{C}$ à l'optimum et $45^{\circ} \mathrm{C}$ au maximum). Les températures horaires sont reconstituées à partir des températures extrêmes journalières et des heures de lever et de coucher du soleil selon deux modèles successifs : sinusoïdal pendant la période diurne et linéaire pendant la période nocturne. Une fonction simple (Fig. 2) permetde calculer un facteur thermique horaire $(F T H)$ et UT = $\Sigma_{h} F T H(h) / 24$. Toutes les phases du développement végétatif sont exprimées en UT. Ce modèle permet aussi de définir un index plastochronique (durée "thermique" nécessaire à l'émergence d'une feuille).

\section{L'unité de développement photothermique (UPT)}

La plupart des stades reproducteurs sont sous la double dépendance de la température et de la photopériode : I'UPT est une "UT nocturne» pondérée par la durée de l'induction florale, (exprimé également en UT nocturnes) pour la photopériode en cours. La variation de ce paramètre avec la photopériode dépend de la variété considérée (Fig. 3).

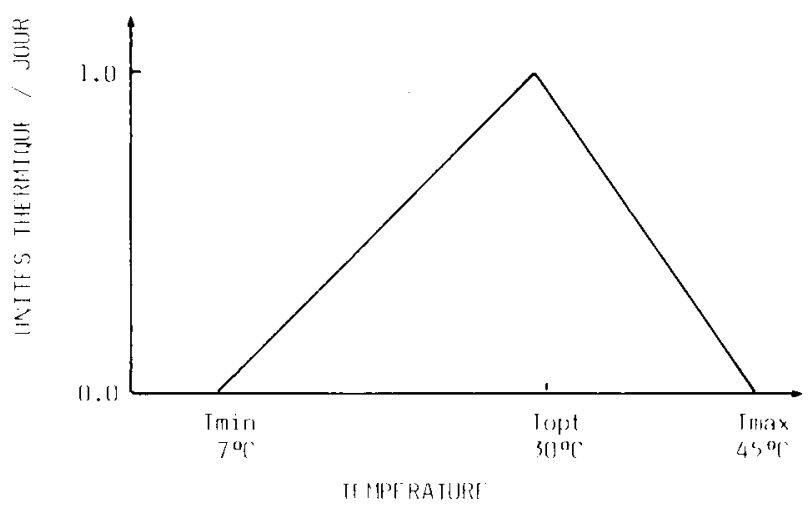

Fig. 2. Calcul de l'unité thermique de développement.

Tableau I. Les données d'entrée du modèle.

\begin{tabular}{|c|c|c|c|}
\hline $\begin{array}{l}\text { Techniques } \\
\text { culturales }\end{array}$ & $\begin{array}{l}\text { Climat } \\
\text { (par jour) }\end{array}$ & $\begin{array}{l}\text { Sol } \\
\text { (Ritchie, 1972, 1985) } \\
\text { (par couche) }\end{array}$ & (ensemble du sol) \\
\hline $\begin{array}{l}\text { - Variété } \\
\text { - Date de semis } \\
\text { - Densité, écartement } \\
\text { - Irrigations }\end{array}$ & $\begin{array}{l}\text { - Températures minimales } \\
\text { et maximales } \\
\text { - Rayonnement global } \\
\text { - Précipitations }\end{array}$ & $\begin{array}{l}\text { - Humidité à la limite } \\
\text { infér. d'extraction d'eau } \\
\text { - Humidité à la limite } \\
\text { de drainage } \\
\text { - Humidité à saturation } \\
\text { - Fonction de densité de } \\
\text { longueur racinaire }\end{array}$ & $\begin{array}{l}\text { - Limite d'évaporation potentielle } \\
\text { du sol nu (création du mulch) } \\
\text { - Albédo du sol nu } \\
\text { - Conductivité hydraulique } \\
\text { - Coefficient de ruissellement } \\
\text { (courbe SCS - USDA) }\end{array}$ \\
\hline
\end{tabular}




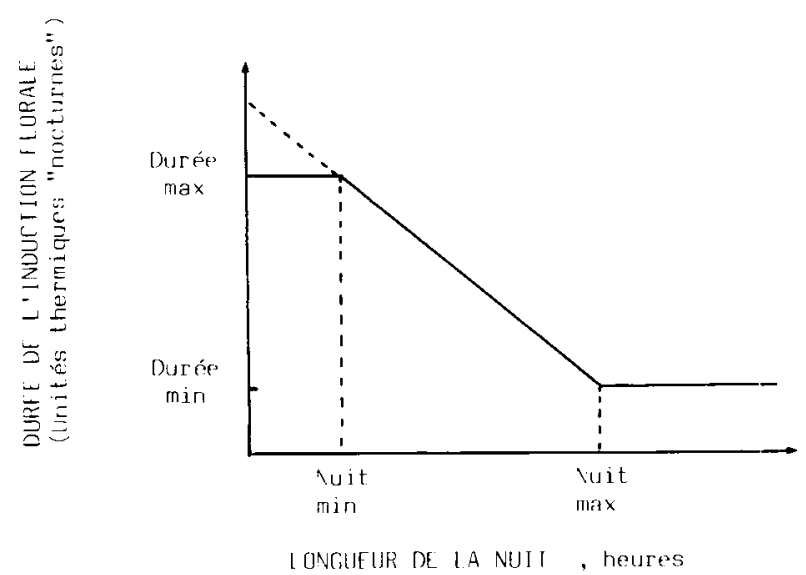

Fig. 3. Calcul de la durée de l'induction florale en fonction de la photopériode.

\section{Le moteur assimilateur}

II modélise la production de matière sèche en prenant en compte photosynthèse brute et respiration. De façon très classique, la photosynthèse brute maximale est calculée en fonction du rayonnement photosynthétiquement actif (PAR) incident (Fig. 4a).

Ce maximum photosynthétique, $P_{G}$ max, peut être réduit par une couverture du sol (indice foliaire) ou une teneur azotée des feuilles non optimales ou, par des stress thermique ou hydrique :

$$
\mathrm{P}_{\mathrm{G}}=\mathrm{P}_{\mathrm{G}} \max \times \underset{\substack{\text { Indice } \\ \text { foliaire }}}{\mathrm{FIF}} \times \underset{\substack{\text { Azote } \\ \text { foliaire }}}{\mathrm{FN}} \times \underset{\substack{\text { Stress } \\ \text { hydrique }}}{\mathrm{FSH}} \times \underset{\substack{\text { Stress } \\ \text { thermique }}}{\mathrm{FT}^{\circ} \mathrm{C}}
$$

Les facteurs de réduction $\mathrm{FIF}, \mathrm{FN}$ et $\mathrm{FT}^{\circ} \mathrm{C}$ varient entre 0 et 1 selon des fonctions ajustées expérimentalement (Figs 4b à 4d); l'indice de stress sera présenté dans la suite de l'article.

La respiration se décompose en une respiration d'entretien (production d'énergie pour la maintenance des membranes cellulaires et de leurs gradients ioniques) et une respiration de croissance (production d'énergie nécessaire au maintien d'une quantité d'enzymes compatible avec la demande due à la croissance : destructions, synthèses). L'intervention de la température sur ces deux types de respiration suit une loi quadratique donnée par McCree (référencé dans Wilkerson et al., 1983).

La photosynthèse nette produit un pool d'assimilats (exprimés en hydrates de carbone : $\mathrm{g} \mathrm{CH}_{2} \mathrm{O} / \mathrm{m}^{2} / \mathrm{j}$ ), qui est réparti, puis converti en matière sèche de composition chimique différente pour chacun des organes de la plante.

\section{Evolution de l'indice foliaire}

En début de période végétative, l'indice foliaire est limité par le potentiel génétique qui fixe la surface maximale
$9 \mathrm{CH}_{2} \mathrm{O} /$ jour $\mathrm{Pg}_{\max }$.

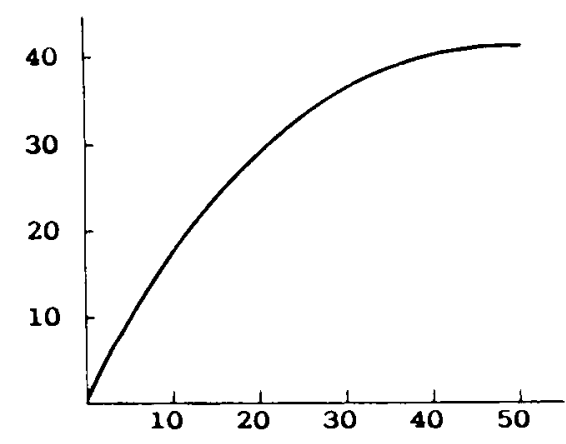

PAK E/m2/jour

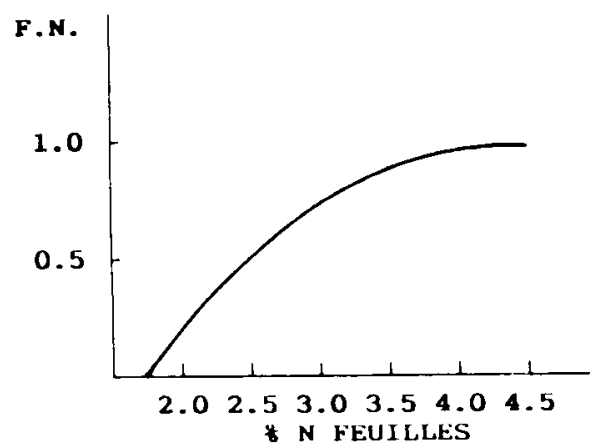

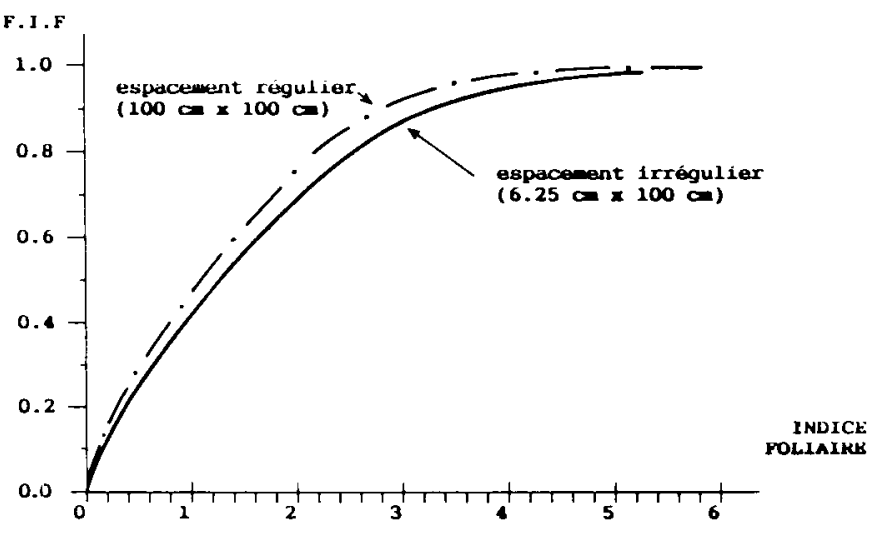

$\boldsymbol{F} r \cdot$

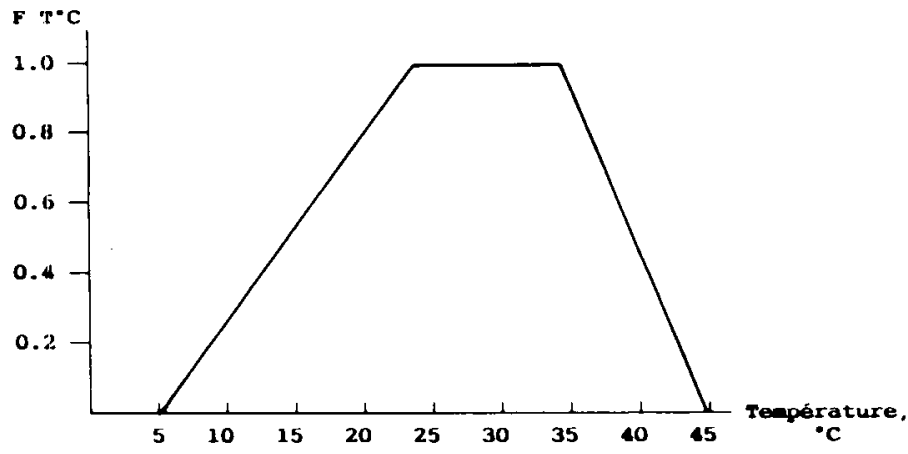

Fig. 4a. Photosynthèse brute maximale, $\mathrm{Pg}_{\max }$, en fonction du $\mathrm{PAR}$; $\boldsymbol{b}$. réduction de $\mathrm{Pg}_{\max }$ par l'indice foliaire; $\mathbf{c}$. réduction de $\mathrm{Pg} \mathrm{g}_{\max }$ par la teneur azotée des feuilles; $d$. réduction de $\mathrm{Pg}_{\max }$ par la température. 
des feuilles et par le rythme de formation des nouveaux nœuuds qui dépend de la température. C'est la surface spécifique (rapport surface/masse) qui permet de convertir la masse en surface. En fin de période végétative, la surface spécifique reste constante jusqu'à ce que les feuilles épaississent, puis elle décroît linéairement pour s'annuler en fin de croissance végétative. Là encore, le stress hydrique peut intervenir.

\section{Elaboration du rendement}

La répartition des assimilats dans les parties végétatives dépend du stade de développement de la plante et du niveau de stress hydrique qui favorise une accumulation de photosynthétats dans les racines. Dès leur apparition, gousses puis graines sont prioritaires quant à leur alimentation en photosynthétats; elles se remplissent jusqu'à un maximum, fonction de la variété, et qui peut être réduit par la température. A partir de la fin de la floraison commencent les remobilisations azotées qui constituent alors l'alimentation première des graines.

La croissance en masse est décrite par les équations suivantes :

$\begin{array}{ll}W=A-\Sigma S & \text { pour l'ensemble du couvert, } \\ W o v=X_{o v} A-S_{o v}-R_{o v} & \begin{array}{l}\text { pour un organe végétatif } \\ \text { feuille, tige ou racine, }\end{array} \\ W g=X_{g} A-R_{g} & \text { pour la gousse, } \\ W g r=X_{g r} A+\Sigma R & \text { pour la graine }\end{array}$

où : $W=$ taux de croissance en masse en $\mathrm{g} / \mathrm{m}^{2}$.jour; $A=$ assimilation nette journalière en $\mathrm{g} / \mathrm{m}^{2}$.jour; $X=$ coefficient de répartition pour l'organe considéré $(\Sigma x=1)$; $\mathrm{S}=$ taux de sénescence en $\mathrm{g} / \mathrm{m}^{2}$.jour; $\mathrm{R}=$ taux des remobilisations azotées en $\mathrm{g} / \mathrm{m}^{2}$.jour.

La vitesse des remobilisations décroît à mesure que la graine se remplit. Le modèle comporte un certain nombre de limitations pour éviter des remobilisations excessives suite à une sécheresse ou lorsque la photosynthèse est faible; de même, la croissance des graines n'est pas indépendante de celle des gousses.

La sénescence foliaire, qui commence dès la formation de la $5^{\circ}$ feuille trifoliée, s'accélère quand interviennent les remobilisations azotées. La sénescence des tiges est proportionnelle à celle des feuilles et toutes deux peuvent être affectées par un stress hydrique. Seul ce dernier facteur peut générer une sénescence racinaire.

La production de gousses peut atteindre un maximum de l'ordre de 180 gousses/m²/jour. Leur avortement est pris en compte lorsque la photosynthèse et la photopériode ne sont pas optimales.

Quand la photosynthèse est limitante, les graines constituent le puits prioritaire, et la production de nouvelles gousses est calculée à partir de la matière sèche restante. Une gousse commence son remplissage 10 jours après fécondation; elle cesse sa croissance 14 jours plus tard. $\mathrm{Si}$, au $10 \mathrm{e}$ jour, les photosynthétats disponibles sont insuffisants (inférieurs à $60 \%$ des besoins des gousses), il y a alors un avortement proportionnel au défaut de photosynthétats. En l'absence de facteur limitant, la gousse se remplit ensuite, et en une seule fois, du nombre moyen de graines par gousse, caractéristique de la variété.
Le module de stress hydrique (Ritchie, 1972,
1985)

II génère deux indices de stress; l'un caractérisant la chute de turgescence (ralentissement des translocations et de la croissance foliaire), l'autre la fermeture des stomates (diminution de la photosynthèse). Besoins en eau de la culture (transpiration maximale) et disponibilité en eau pour le système racinaire sont calculés en parallèle et c'est le rapport, disponibilité/transpiration maximale, qui définit les indices de stress. En fonction de l'indice foliaire, l'ETP est décomposée en évaporation potentielle du sol et transpiration maximale de la culture. Parallèlement, un modèle de croissance racinaire crée une densité de longueur racinaire, qui peut exploiter la réserve en eau du sol, définissant ainsi la disponibilité.

Dans SOYGRO, le sol joue uniquement un rôle de réservoir hydrique et n'intervient donc que lorsqu'il y a déficit hydrique. L'alimentation minérale de la plante est considérée comme optimale.

\section{Analyse de sensibilité variétale du modèle SOYGRO}

Le modèle comporte 87 paramètres, qui ne sont pas tous indépendants les uns des autres. II y a des paramètres d'ajustement (exemple des paramètres définissant les courbes des Figures 4) et des paramètres mesurés. Ce sont ces derniers qui forment l'essentiel des 17 paramètres liés à la variété.

Parmi ces paramètres variétaux, certains définissent le développement de la plante, d'autres en gèrent la croissance (élaboration du feuillage et du rendement). Les premiers caractérisent le groupe de précocité, pour un même type de croissance (Ecochard, 1986); tandis que les seconds dépendent de l'ensemble des éléments du potentiel de production variétal. La sensibilité du modèle aux paramètres variétaux indique le degré de précision nécessaire pour chacun d'eux et par suite, l'intérêt de mettre en place des expérimentations spécifiques pour les mesurer.

Dans une première approche, nous avons choisi de sélectionner des conditions d'alimentation en eau non limitantes et donc nous n'avons pas pris en compte le module de stress hydrique. L'étude entreprise permet donc de tester la sensibilité du modèle sur les aspects phénologie, croissance et élaboration du rendement en condition de cultures optimales.

\section{Conditions générales}

La variété Waine (groupe de précocité III) utilisée dans SOYGRO a servi de référence témoin. Nous avons fait varier la valeur des paramètres dans une fourchette de $\pm 10 \%$ pour faire des simulations, en 2 sites différents, Montélimar et Toulouse, et pour 2 années climatiquement différentes : 1984 et 1986 (l'année 1984 étant plus froide que l'année 1986). S'il y a un effet site, ce ne peut être qu'un effet climatique et non pédologique puisque le sol n'intervient que dans le module stress hydrique qui n'est pas pris en compte. Les résultats sont expri- 
més par la variation du rendement en pourcentage par rapport au témoin (Tableau II).

Il est possible, à la lecture du tableau, d'affirmer que, les paramètres qui ont le plus d'impact sur le rendement sont d'une part, ceux caractérisant la phénologie liée à la reproduction, d'autre part ceux traduisant l'élaboration du rendement, alors que l'effet de la mise en place du feuillage semble peu important.

\section{Les paramètres phénologiques}

Les trois paramètres phénologiques jouent sur la durée floraison-maturité. La durée de la maturation, qui sépare les stades R7 (maturité physiologique) et R8 (maturité de récolte), n'a aucune influence sur le rendement; ce qui s'explique par le fait qu'au début de cette période (R7), la quasi-totalité de la matière sèche est en place dans les graines (Salado-Navarro et al., 1985). Par contre, le seuil photopériodique et la durée floraison (R1)-maturité (R7), qui servent à calculer la durée photothermique de cette phase reproductrice ont un poids marqué sur le rendement. Une variation du seuil photopériodique peut la déplacer et, le glissement qui en résulte, sur des périodes où la durée d'éclairement journalière est plus longue, permet un supplément de photosynthèse, puis de rendement qui peut, certaines années, compenser l'effet du raccourcissement du cycle.

\section{Les paramètres d'élaboration du rendement}

Le rendement se décompose classiquement en : nbre gousses $/ \mathrm{m}^{2} \times$ nbre graines/gousse $\times$ poids de la graine. Or, ces 3 composantes dépendent d'un certain nombre de paramètres introduits dans le modèle : nombre maximum de gousses initiées, nombre moyen de graines par gousse et croissance de la graine. L'influence de ces 3 paramètres est très voisine quels que soient le site et l'année.

Par ailleurs, une augmentation du potentiel de croissance des cosses provoque une baisse de rendement, d'autant plus importante que l'année est plus chaude

Tableau II. Résultats de l'analyse de sensibilité variétale.

\begin{tabular}{|c|c|c|c|c|c|c|c|c|}
\hline \multirow[t]{5}{*}{ Paramètres } & \multicolumn{8}{|c|}{ Rendement - Rendement témoin } \\
\hline & \multicolumn{8}{|c|}{ Rendement témoin } \\
\hline & \multicolumn{4}{|c|}{$-10 \%$ (sauf pour 1) } & \multicolumn{4}{|c|}{$+10 \%$} \\
\hline & & & & Iont. & & ul. & & \\
\hline & 1984 & 1986 & 1984 & 1986 & 1984 & 1986 & 1984 & 1986 \\
\hline \multicolumn{9}{|l|}{ Phénologie } \\
\hline $\begin{array}{l}\text { 1. Seuil photopériodique } \\
(-5 \%) \text { (heures) }\end{array}$ & 5,9 & $-2,1$ & 17,7 & -10 & 1 & 1 & 1 & 1 \\
\hline $\begin{array}{l}\text { 2. Durée R1-R73 } \\
\text { cycle reproducteur } \\
\text { (u. photothermique) }\end{array}$ & $-15,7$ & $-10,9$ & -8 & $-12,7$ & 9,6 & 17,3 & 11,1 & 15,3 \\
\hline $\begin{array}{l}\text { 3. Durée R7-R8 } \\
\text { maturation (u. thermique) }\end{array}$ & 0 & 0 & 0 & 0 & 0 & 0 & 0 & 0 \\
\hline \multicolumn{9}{|l|}{ Elaboration du rendement } \\
\hline $\begin{array}{l}\text { 4. Nombre maxi gousses } \\
\text { initiées par } \mathrm{m}^{2} \text { et par jour }\end{array}$ & $-3,3$ & $-3,2$ & $-3,4$ & $-2,2$ & 3 & 3,1 & 3,4 & 1,6 \\
\hline $\begin{array}{l}\text { 5. Nombre moyen } \\
\text { grains/gousses }\end{array}$ & $-4,3$ & $-4,4$ & -4 & $-3,4$ & 3,7 & 3,8 & 4 & 2,8 \\
\hline $\begin{array}{l}\text { 6. Croissance de la cosse } \\
\text { (mg/jour) }\end{array}$ & 0,5 & 0,9 & 0,2 & 1,6 & $-0,2$ & $-1,1$ & $-0,1$ & $-1,5$ \\
\hline $\begin{array}{l}\text { 7. Croissance de la graine } \\
\text { (mg/jour) }\end{array}$ & $-4,3$ & $-4,4$ & -4 & $-3,4$ & 3,6 & 3,8 & 4 & 2,8 \\
\hline \multicolumn{9}{|l|}{ Elaboration de l'IF } \\
\hline $\begin{array}{l}\text { 8. Taille feuille } \\
\left(\mathrm{cm}^{2} / \mathrm{g}\right) \text { standard2 }\end{array}$ & $-0,1$ & $-0,1$ & $-0,2$ & $-0,09$ & 0,1 & 0,08 & 0,2 & 0,05 \\
\hline $\begin{array}{l}\text { 9. Surface foliaire spécifique } \\
\left(\mathrm{cm}^{2} / \mathrm{g}\right)\end{array}$ & $-0,6$ & $-0,6$ & $-0,5$ & $-0,4$ & 0,4 & 0,3 & 0,3 & 0,2 \\
\hline $\begin{array}{l}\text { 10. Index plastochronique } \\
\text { (u. thermique) }\end{array}$ & $-0,3$ & $-0,2$ & $-0,3$ & $-0,05$ & 0,2 & 0,1 & 0,2 & 0,08 \\
\hline
\end{tabular}

\footnotetext{
${ }^{1}$ Seuil n'existant pas sous nos latitudes; ${ }^{2}$ mesurées sur la feuille adulte du $8^{8}$ au $10^{\ominus}$ nœud; ${ }^{3}$ stades de Fehr et Caviness (1971).
} 
(comparaison 1984-1986). Ce qui s'explique par le fait que les cosses vont utiliser davantage de photosynthétats au détriment des graines. Néanmoins, ce phénomène restera limité par l'ordre de priorité de la répartition, (en premier la graine, et en second la cosse).

Toutefois, il semblerait que les deux paramètres de croissance pondérale ne soient pas totalement indépendants; la croissance pondérale potentielle de la cosse est voisine du double de celle de la graine (Wilkerson et al., 1985).

\section{Les paramètres de mise en place de l'appareil foliaire}

La faible variation du rendement induite par une modification de $\pm 10 \%$ de la taille, de la surface spécifique des feuilles ou de l'index plastochronique confirme les observations selon lesquelles un indice foliaire de 4,5 correspond à un appareil assimilateur suffisant pour alimenter les parties végétatives et les gousses (Blanchet et Gelfi, 1978).

\section{Remarques sur les différences entre sites et années}

Les simulations du Tableau II soulignent la différence de variabilité interannuelle entre Montélimar et Toulouse vis-à-vis du rendement : les écarts entre 1984 et 1986 sont plus accusés à Montélimar. D'autre part, en 1986, la variation de rendement induite par la variation de l'un quelconque des paramètres liés au rendement est globalement plus faible que celle de 1984; cela laisse à penser que le modèle prend en compte des phénomènes de compensation en année chaude.

\section{Calibration et validation du modèle pour des variétés de précocité I et II en l'absence de stress hydrique}

\section{Conditions générales}

Pour réaliser la calibration, nous disposions de résultats d'essais (correspondant aux paramètres variétaux du modèle) conduits de 1984 à 1987 à Montélimar (CETIOM), à Toulouse (INRA), à Nîmes (Compagnie du Bas-Rhône) ou à Padoue en Italie (Université), ainsi que les données climatiques correspondantes.

Parmi les variétés choisies, deux sont à croissance indéterminée, Weber (groupe de précocité I), et Kingsoy (groupe de précocité II) et, une à croissance déterminée, INRA 172-16 (groupe de précocité I)

Pour la validation, nos observations de stades de développement et de composantes du rendement, complétées par celles données par Blanchet et al. (1986, 1987), et Baret (communication personnelle), ont été comparées aux valeurs simulées par SOYGRO dans des conditions culturales similaires : mêmes dates et densités de semis, absence de stress hydrique.

\section{La phénologie}

Les Tableaux III et IV, montrent que le déroulement du cycle est simulé de façon satisfaisante avec une erreur ${ }^{\star}$ de 5 jours à la floraison et de 4 jours en fin de cycle.

\section{Les composantes du rendement}

Le Tableau $V$ permet de comparer les performances agronomiques simulées et observées d'une variété à croissance indéterminée (Kingsoy) et d'une variété à croissance déterminée (Inra 172-16). II montre que les particularités des variétés à croissance déterminée et indéterminée sont respectées : production de gousses plus élevée chez Kingsoy avec, en contrepartie, un remplissage moindre que chez 172-16, tant au niveau du nombre de graines que de leur poids (Vidal et al., 1985; Blanchet et al., 1986; Blanchet et al., 1987). Toutefois le poids de 1000 graines semble surestimé.

Une explication possible pourrait être le mode de calcul du rendement et de ses composantes. En effet, dans SOYGRO, le rendement résulte du calcul du poids de graines produites par $\mathrm{m}^{2}$, ultérieurement diminué d'un taux fixe de $17 \%$, comptabilisant ainsi les pertes à la récolte. Le poids de graines dépend des assimilats disponibles; il est limité par le nombre de gousses mises en place. A partir de ces deux éléments calculés par le modèle, il est possible de reconstituer le poids de 1000 graines connaissant, par ailleurs, le nombre de graines par gousse (paramètre variétal). Ainsi calculées, les composantes du rendement (nombre de gousses $/ \mathrm{m}^{2 *}$ nombre de graines/gousse ${ }^{\star}$ poids de 1000 graines) ne tiennent pas compte des pertes.

Dans la pratique, il est fréquent que l'une des composantes du rendement soit ajustée au rendement «moissonneuse" et donc intègre les pertes (c'est le cas des données observées du Tableau V).

A Nîmes (Compagnie du Bas-Rhône) en 1987, les pertes variaient de 9 à $20 \%$ tandis que Scott et Aldrich (1970) parlent de 5 à 19\% de pertes dues essentiellement à la mécanisation de la moisson. Les auteurs de SOYGRO ont donc choisi un niveau élevé de pertes.

Par ailleurs, dans SOYGRO, la variabilité du rendement n'est contrôlée que par deux des trois composantes (nombre de gousses par $\mathrm{m}^{2}$ et poids de 1000 graines); le nombre de graines par gousse étant fixé variétalement. Or cette composante peut, comme les autres composantes, être sensible aux stress et en particulier au stress hydrique; néanmoins, parmi les trois composantes, ce serait la plus stable pour une variété donnée (Puech et Bouniols, 1986).

Compte tenu du mode de calcul du rendement et de la fixation du nombre de graines par gousse, la comparaison des valeurs simulées et observées du Tableau $V$ semble délicate.

L'erreur de simulation des rendements (Tableau VI) est de 3,2 q/ha, ce qui indique un comportement très satisfaisent de SOYGRO compte tenu des limitations précédemment évoquées.

\footnotetext{
* L'erreur calculée est la racine carrée de l'erreur quadratique moyenne.
} 
Tableau III. Comparaison des dates de floraison observées et simulées.

\begin{tabular}{|c|c|c|c|c|c|c|}
\hline & & 1985 & $\begin{array}{l}\text { Toulouse } \\
1986\end{array}$ & 1987 & $\begin{array}{l}\text { Nîmes } \\
1987\end{array}$ & $\begin{array}{l}\text { Avignon } \\
1987\end{array}$ \\
\hline $\begin{array}{l}\text { Weber } \\
\text { Kingsoy } \\
172-16\end{array}$ & $\begin{array}{l}\text { Obs. } \\
\text { Sim. } \\
\text { Obs. } \\
\text { Sim. } \\
\text { Obs. } \\
\text { Sim. }\end{array}$ & $\begin{array}{l}02 \text { juillet } \\
27 \text { juin } \\
06 \text { juillet } \\
03 \text { juillet }\end{array}$ & $\begin{array}{l}25 \text { juin } \\
24 \text { juin } \\
02 \text { juillet } \\
03 \text { juillet } \\
27 \text { juin } \\
28 \text { juin }\end{array}$ & $\begin{array}{l}29 \text { juin } \\
21 \text { juin } \\
30 \text { juin } \\
23 \text { juin }\end{array}$ & $\begin{array}{l}21 \text { juillet } \\
18 \text { juillet }\end{array}$ & $\begin{array}{l}05 \text { août } \\
29 \text { juillet }\end{array}$ \\
\hline
\end{tabular}

Tableau IV. Comparaison des dates de fin de cycle (R7 ou R8) observées et simulées.

\begin{tabular}{|c|c|c|c|c|c|}
\hline & & 1985 & $\begin{array}{l}\text { Toulouse } \\
1986\end{array}$ & 1987 & $\begin{array}{l}\text { Avignon } \\
1987\end{array}$ \\
\hline $\begin{array}{l}\text { Weber } \\
\text { Kingsoy } \\
172-16\end{array}$ & $\begin{array}{l}\text { Obs. } \\
\text { Sim } \\
\text { Obs. } \\
\text { Sim. } \\
\text { Obs. } \\
\text { Sim. }\end{array}$ & $\begin{array}{l}15 \text { septembre } \\
18 \text { septembre } \\
15 \text { octobre } \\
12 \text { octobre }\end{array}$ & $\begin{array}{l}01 \text { octobre } \\
27 \text { septembre } \\
08 \text { octobre } \\
12 \text { octobre } \\
01 \text { octobre } \\
03 \text { octobre }\end{array}$ & $\begin{array}{l}15 \text { septembre } \\
12 \text { septembre }\end{array}$ & $\begin{array}{l}20 \text { septembre } \\
25 \text { septembre }\end{array}$ \\
\hline
\end{tabular}

Tableau V. Comparaison des paramètres agronomiques simulés et observés de Kingsoy et INRA 172-16.

\begin{tabular}{|c|c|c|c|c|c|c|}
\hline $\begin{array}{l}\text { Paramètres } \\
\text { agronomiques }\end{array}$ & $\begin{array}{l}\text { Kingsc } \\
\text { Obs. }\end{array}$ & $\begin{array}{l}\text { oy. } 1985 \\
\text { Sim. }\end{array}$ & $\begin{array}{c}\text { 1986) } \\
\text { Diff. } \\
(\%)\end{array}$ & $\begin{array}{l}172-16 \\
\text { Obs. }\end{array}$ & $\begin{array}{l}1985 \\
\text { Sim. }\end{array}$ & $\begin{array}{c}1987) \\
\text { Diff. } \\
(\%)\end{array}$ \\
\hline $\begin{array}{l}\text { Gousses/m² } \\
\text { Poids } 1000 \mathrm{~g} \\
\text { Nb. gr./gousse } \\
\text { Ind. récolte } \\
\text { Nb. nœeuds } \\
\text { Production en } \mathrm{q} / \mathrm{ha} \\
\text { Rendement } \\
\text { moissonneuse en } \mathrm{q} / \mathrm{ha}\end{array}$ & $\begin{array}{l}1183 \\
186 \\
1,92 \\
0,46 \\
19,5\end{array}$ & $\begin{array}{l}1237 \\
204 \\
1,94 \\
0,51 \\
21 \\
48,9 \\
40,6\end{array}$ & $\begin{array}{l}+4,6 \\
+9,7 \\
+1 \\
+10,9 \\
+7,7\end{array}$ & $\begin{array}{l}1060 \\
188 \\
2,21 \\
0,53 \\
11,2\end{array}$ & $\begin{array}{l}932 \\
270 \\
2,14 \\
0,58 \\
9,2 \\
53,8 \\
44,7\end{array}$ & $\begin{array}{l}-12,1 \\
+43,6 \\
-3,2 \\
+11,3 \\
-17,8\end{array}$ \\
\hline
\end{tabular}

\section{Discussion et Conclusion}

SOYGRO simule, par l'enchainement de modules spécifiques, la phénologie et les principales fonctions physiologiques de la plante : photosynthèse, respiration, répartition des assimilats dans les différents organes, sénescence, au pas de temps journalier et à l'échelle du couvert. Chaque jour, le modèle génère des données permettant de contrôler la culture des points de vue physiologique et agronomique.

Les résultats du travail exposé ici montrent l'intérêt du modèle SOYGRO pour simuler le déroulement du cycle cultural et l'élaboration du rende- 
Tableau VI. Comparaison des rendements observés et simulés en q/ha.

\begin{tabular}{|c|c|c|c|c|c|c|}
\hline & & 1985 & $\begin{array}{c}\text { Toulous } \\
1986\end{array}$ & ${ }_{1987}$ & $\begin{array}{l}\text { Nimes } \\
1987\end{array}$ & $\begin{array}{l}\text { Avignon } \\
1987\end{array}$ \\
\hline Weber & $\begin{array}{l}\text { Obs. } \\
\text { Sim. }\end{array}$ & $\begin{array}{l}40,9 \\
45,4\end{array}$ & $\begin{array}{l}45,5 \\
40,7\end{array}$ & $\begin{array}{l}39,3 \\
39,5\end{array}$ & $\begin{array}{l}35,2 \\
36,2\end{array}$ & $\begin{array}{l}36,8 \\
36,8\end{array}$ \\
\hline Kingsoy & $\begin{array}{l}\text { Obs. } \\
\text { Sim. }\end{array}$ & $\begin{array}{l}41,2 \\
42,5\end{array}$ & $\begin{array}{l}44,1 \\
38,7\end{array}$ & & & \\
\hline $172-16$ & $\begin{array}{l}\text { Obs. } \\
\text { Sim. }\end{array}$ & $\begin{array}{l}42,7 \\
46,4\end{array}$ & $\begin{array}{l}44,7 \\
43,3\end{array}$ & $\begin{array}{l}41,6 \\
44,6\end{array}$ & & \\
\hline
\end{tabular}

ment pour différentes variétés. II est en particulier intéressant de pouvoir comparer les performances des variétés à croissance indéterminée avec celles des variétés à croissance déterminée.

Dans les conditions climatiques du Sud de l'Europe, l'adaptation du modèle repose sur les paramètres variétaux qui sont accessibles à l'expérimentation. En effet, l'avantage d'un modèle mécaniste est qu'il tente de réduire au minimum les paramètres d'ajustement au profit de paramètres mesurables. Cependant, la limite entre paramètres variétaux et paramètres indépendants de la variété reste liée à la diversité des variétés testées pour la mise au point du modèle. Ainsi, la courbe de réponse de la photosynthèse à la température (Figure 4 d) pourrait devenir une courbe variétale si l'on s'adressait à une gamme étendue de précocités.

Néanmoins, il faut souligner les limites du modèle : les caractéristiques de la nutrition azotée du soja sont ignorées (besoins élevés, alimentation mixte par fixation symbiotique et absorption d'azote combiné). En réalité, les nodosités jouent à la fois un rôle de "puits" pour les photosynthétats et de "source" pour les composés azotés. Par ailleurs, l'extrapolation du modèle à des zones plus septentrionales peut nécessiter des adaptations à l'influence des températures fraîches; cet effet interviendrait à deux niveaux :

- au niveau photosynthétique : la courbe de réponse de la photosynthèse brute à la température a été ajustée avec des températures moyennes dépassant $15^{\circ} \mathrm{C}$, ce qui a pour conséquence de simuler un arrêt de la fonction assimilatrice pour des températures moyennes en deçà de $5^{\circ} \mathrm{C}$. Or cette limite semble quelque peu élevée pour des variétés des groupes 0 et 00 (Planchon, communication personnelle);

- au niveau de l'avortement des fleurs, phénomène non pris en compte par le modèle : une période préflorale froide peut avoir des incidences sur le nombre d'organes reproducteurs en production (Pigeaire, 1984).

Dans le modèle, c'est essentiellement le poids de 1000 graines qui détermine la variabilité du rendement. Or, le rendement s'explique le plus souvent par le nombre de gousses $/ \mathrm{m}^{2}$ (Puech et Bouniols, 1986; Pigeaire, 1984). En effet, le poids de 1000 graines ne peut dépasser un certain seuil pour une variété donnée (Prudon et Merrien, 1986). En conséquence, il faudrait peut-être envisager de plafonner le poids de 1000 graines pour chaque variété.

SOYGRO est un modèle centré sur le cycle de la culture du soja : la simulation commence au semis et s'achève à la récolte, mais elle ne donne aucune indication sur le choix de la date de semis (limitations liées à la température dans le sol ou à son état hydrique).

Notre étude a montré que SOYGRO simule correctement des variétés à croissance déterminée et indéterminées dans des conditions méridionales et en l'absence de stress hydrique. Cela constitue une première étape dans l'utilisation de ce modèle pour prévoir le comportement agronomique de différentes variétés de soja. La prise en compte du stress hydrique, qui nécessite de connaître avec précision le sol, est, dans cette optique, une seconde étape qui permettra de comprendre les contraintes exercées par le milieu.

On pourra, alors, évaluer les potentialités d'une variété de soja dans une région donnée compte tenu de son climat, de son sol et de la mise en œuvre de certaines techniques culturales.

\section{Remerciements}

Ce travail a été réalisé avec l'appui de la station de bioclimatologie-STEFCE de l'INRA-Avignon et avec l'aide du CETIOM. Nous remercions B. Seguin pour sa contribution appréciée à l'amélioration du manuscrit. 


\section{Références}

Blanchet R. \& Gelfi N. (1978) Relations entre développement foliaire, transpiration et production chez le soja/cv. Amsoy 71 et Hodgson. Ann. Agron. 29, 223-242 Blanchet R., Bouniols A., Constants S., Gelfi N., Marthy J.R. \& Puech J. (1986) Biologie et formation du rendement de sojas déterminés et indéterminés en bonnes conditions culturales. Eurosoya 4, 10-17

Blanchet R., Hugon C. Bouniols A. \& Gelfi N. (1987) Réponse comparée de sojas à croissance déterminée ou non à différentes ressources hydriques. Eurosoya 5 , 22-30

Ecochard R. (1986) La sensibilité du soja à la photopériode et à la thermopériode. In : Le Soja : Physiologie de la Plante et Adaptation aux Conditions Françaises. Suppl. Inf. Techn. CETIOM, pp. 91-98

Ecochard R., Denuc M. \& Aussel P. (1978) Développement physique et productivité du soja : étude variétale. Ann. Amélior. Plant. (Paris) 28, 4, 351-370

Fehr W.R., Caviness C.E., Burmood D.T. \& Pennington J.S. (1971) Stage of development descriptions for soybeans Glycine max. Crop Sci. 11, 929-931

Hodges T. \& French V. (1985) Soyphen: soybean growth stages modeled from temperature, daylength and water availibility. Agron. J. 1, 77, 500-505

Hodges T., Botner D., Sakamoto C. \& Hays Haug J. (1987) Using the CERES-Maiis model to estimate production for the US cornbelt. Agric. For. Metéorol. 40, 293-303

Meyer G.E., Curry R.B., Streeter J.G. \& Baker C.H. (1981) Simulation of reproductive processes and senescence in indeterminate soybeans. Trans. Am. Soc. Agric. Eng. 421-435

Pigeaire A. (1984) Elaboration des composantes du rendement chez le soja de type indéterminé. Thèse Toulouse

Prudon E. \& Merrien A. (1986) Composantes du rendement chez le soja : variabilité génétique et effets du milieu. In : Le Soja : Physiologie de la Plante et Adaptation aux Conditions Françaises. Suppl. Irif. Techn. CETIOM, pp. 113-116

Puech J. \& Bouniols A. (1986) Floraison, fructification et composantes du rendement du soja : réflexion pour l'ob- tention de hauts rendements. In : Le Soja : Physiologie de la Plante et Adaptation aux Conditions Françaises. Suppl. Inf. Techn. CETIOM, pp. 58-72

Ritchie J.T. (1972) Model for predicting evaporation from a row crop with incomplete cover. Water Resour. Res. 8, 5, 1204-1213

Ritchie J.T. (1985) A user-oriented model of the soil water balance in wheat. In : Wheat Growth and Modeling (E. Fry and T.K. Atkin, eds.), Plenum Publishing Corporation, NATO-ASI Series, pp. 293-305

Salado-Navarro U.R., Sinclair T.R. \& Hinson K. (1985) Comparisons among effective filling period, reproductive period duration and R5 to R7 in determinate soybeans. Crop. Sci. 25, 1050-1054

Scott W.O. \& Aldrich S.R. (1970) Modern Soybean Production (B. Barksdale, ed.), S \& A Publications, Champaign, Illinois, USA

Seddigh M. \& Jollif G.D. (1984) Night temperature effects on morphology, phenology, yield and yield components of indeterminate field grown soybean. Agron. $J$. $76,824-828$

Shanmugasundaram S. (1978) Photoperiod and critical duration for flower induction in soybean. Crop Sci. 18, 598-601

Sinclair T.R. (1986) Water and nitrogen limitations in soybean grain production. 1 : Model development. Field Crops. Res. 15, 125-141

Vidal A., Hanafiah T.O., Meynie S., Poux G. \& Vares M. (1985) Influence du type de croissance sur la morphologie et la floraison du soja. Eurosoya 3, 62-70

Whisler F.D., Acock B., Baker D.N., Fye R.E., Hodges H.F., Lambert J.R., Lemmon H.E., McKinion J.M. \& Reddy V.R. (1986) Crop simulation models in agronomic systems. Adv. Agron. 40, 141-209

Wilkerson G.G., Jones J.W., Boote K.J., Ingram K.T. \& Mishoe J.W. (1983) Modeling soybean growth for crop management. Trans. Am. Soc. Agric. Eng. 26, 63-73

Wilkerson G.G., Jones JW., Boote K.J. \& Mishoe J.W. (1985) SOYGRO Version 5.0 : Soybean Crop Growth and Yield Model. Internal Report, Gainsville University, Floride, USA

Williams J.R., Jones C.A. \& Dyke P.T. (1984) A modeling approach to determining the relationship between erosion and soil productivity. Trans. Am. Soc. Agric. Eng. 27, 129-144 